\title{
Electrochemical investigations of aluminium alloys tribocorrosion
}

\author{
V. I. Pokhmurskii, I. M. Zin,* H. V. Pokhmurska and V. A. Vynar \\ G.V.Karpenko Physico-Mechanical Institute of National Academy of Sciences of Ukraine, \\ 79603 Lviv, Ukraine \\ *E-mail: zin@ipm.lviv.ua
}

\begin{abstract}
Electrochemical methods based on corrosion potential and polarization current measurements are widely used in tribocorrosion research. However, most equipment types allow recording of integrated electrochemical parameters of metals during tribocorrosion, but little attention is paid to local processes that occur on friction surfaces. It was proposed to use a capillary probe as a reference electrode, which reduces the effect of cathodic sites on sample tribopotential and allows measurements in low-conductivity solutions. The study was carried out on an example of the widely used AA2024 alloy, and may relate to structural aluminum alloys. It was shown that the difference between tribopotential values of the alloy measured by classic electrochemical technique and capillary probe may be up to $0.6 \mathrm{~V}$ in a study in glycerin. It was established that correlation exists between the current of alloy polarization at the corrosion potential in distilled water and the volume of worn material. The empirical relationship found for a ball-on-flat tribometer with reversible sliding provides a possibility to estimate the amount of worn material based on polarization currents. This method may also be promising in studies on the efficiency of corrosion inhibitors on mechanically activated surface of passive metal.
\end{abstract}

Keywords: aluminium alloy, tribocorrosion, corrosion potential, friction coefficient, polarization current, capillary microprobe.

Received: January 29, 2014; in revised form: March 18, 2014. doi: 10.17675/2305-6894-2014-3-2-129-136

\section{Introduction}

Tribocorrosion is irreversible transformation of surface and subsurface layers of metals and alloys caused by physical, chemical and mechanical interactions during their frictional contacts in corrosion environments [1-4]. It includes a number of synergistic effects between mechanical, chemical and electrochemical processes due to which material losses from tribocorrosion are much higher than a simple sum of the mechanical wear and corrosion losses determined separately. This kind of destruction is widespread in friction units of food, mining, processing, chemical and many others industries.

Electrochemical methods are widely used to study tribocorrosion processes [5-7]. However, interpretation of their results is not always correct because of inadequate methodological approaches. In this paper we describe a method of electrochemical investigation of wear track on passive metal surface during friction interactions using a 
reference electrode microprobe and a study of polarization current changes associated with sample material loss due to electrochemical processes. This method can also be used for corrosion inhibition study of mechanically activated (rubbing) surface of passive metal. The investigations were carried out on commonly used AA 2024 aluminium alloy.

\section{Experimental procedure}

Tribocorrosion tests of metal samples in environments with different aggressiveness and conductivity were performed on a special self-made tribometer using linear reciprocating ball-on-flat geometry (Fig. 1). Construction of the unit eliminates the unregulated movement of the contact pair during the test, which is very important for conducting this type of experiment, especially in determination of geometrical parameters of the wear track. It consists of a frame (1) and a reciprocating stage (2). The stage is driven by an electric motor through a worm gear. Bearings (4) which provide straightforward movement of the stage are attached to the frame. A flat alloy sample (10) is pressed to the stage (2) by a corrosion cell (3) fastened to the stage through a rubber seal.

The sample was loaded by a weight (7) placed on the top of indenter support (8), which was mounted in the balance-beam (9). The balance-beam is placed in the right position by use of a level ( 6 ) to ensure perpendicularity of the indenter to the surface of the sample (10) upon applying a normal load. Strain sensors (11) were fixed to the indenter support and recorded the moment of friction forces during the tests. The friction coefficient between the ceramic ball and the aluminium alloy is calculated using the values of the moment.

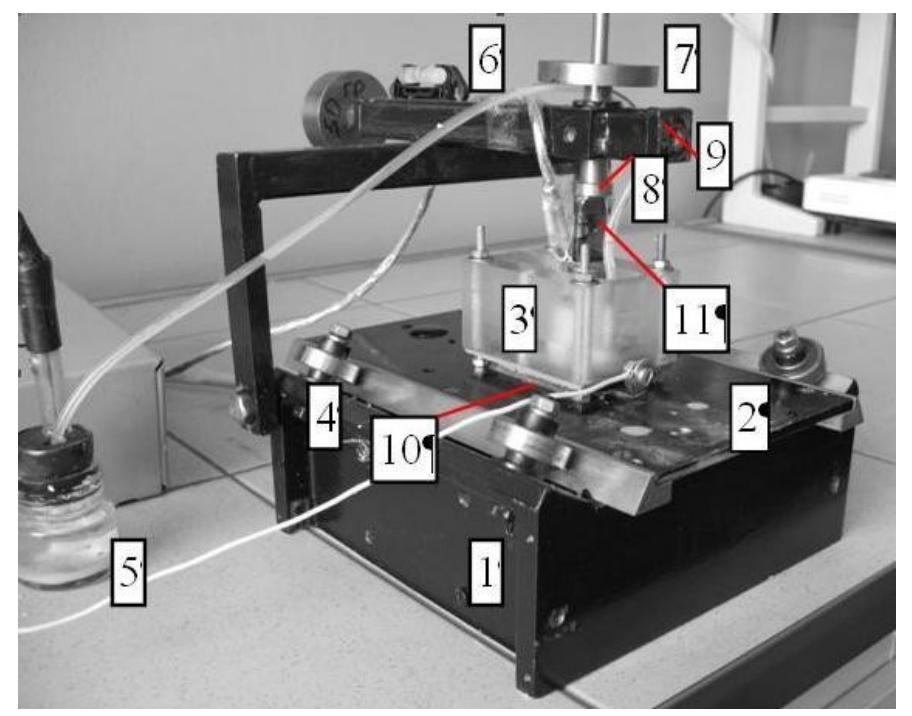

Figure 1. Equipment for tribocorrosin testing: 1 - frame; 2 - reciprocating stage; 3 - corrosion cell, 4 - bearings; 5 - Ag/AgCl reference electrode; 6 - level; 7 - load; 8 -indentor support; 9 - balance-beam; 10 - sample; 11 - strain sensor. 
Test samples $(50 \times 40 \times 5 \mathrm{~mm})$ were made of aluminium alloy AA2024. Tribocorrosion experiments were carried out in environments of different conductivity: glycerin, distilled water and $3 \% \mathrm{NaCl}$ solution. A corundum ball with $9 \mathrm{~mm}$ diameter was used as an indenter. Changes in friction moment and corrosion potential of aluminium alloy sample were recorded simultaneously. Corrosion potential was measured using a capillary microprobe of $200 \mu \mathrm{m}$ internal diameter connected to saturated $\mathrm{Ag} / \mathrm{AgCl}$ reference electrode. Platinum wire served as the counter electrode. Changes in the parameters were registered at measurement intervals of $0.25 \mathrm{~s}$ using an analog-digital converter and then transferred to a PC.

Topography of the wear surface and analysis of tribocorrosion products were studied by a scanning electron microscope EVO-40XVP (Carl Zeiss).

\section{Experimental results and discussion}

\subsection{Use of a capillary probe to study the corrosion potential changes during tribocorrosion tests}

Tribocorrosion process development can be estimated on the basis of corrosion potential changes of a metal during wear in a corrosive solution [5]. Of particular interest are local changes in corrosion potential on wear track, because the electrochemical state of a metal in the contact zone depends on surface film destruction, as well as on contact stresses.

The potential measured during reciprocating friction of a metal has a compromise value $E_{\text {mix }}$ between the potentials of the loaded and unloaded zones of the sample. It was proposed to eliminate the influence of non-worn surface by using a capillary microprobe for measurements of the electrode potential of the track area (Fig. 2). The capillary is made of a polymer material and filled with agar-agar gel saturated with $\mathrm{KCl}$. The capillary inner diameter was about $1 \mathrm{~mm}$ and the diameter of its working section was $200 \mu \mathrm{m}$. The capillary was placed as close as possible to the friction zone (Fig. 2) and moved behind the indentor in one direction and in front of it in the opposite direction.

The values of the electrode potential measured in poorly conductive solution with the microprobe method differ from those obtained by the classical method. Tests of alloy AA2024 samples in glycerine, whose conductivity is about $8 \cdot 10^{-6} \mathrm{Ohm} / \mathrm{m}$, have determined a significant difference in potential values. The values measured with a microprobe are more negative by about $600 \mathrm{mV}$ (Fig. 3a).

In $3 \% \mathrm{NaCl}$ solution with conductivity $\Omega=4.7 \mathrm{~S} / \mathrm{m}$, the difference is insignificant, i.e., $\sim 40 \mathrm{mV}$ (Fig. 3b), and another pattern of electrode potential change was observed. Its sudden shift towards more negative values may indicate activation of the wear track surface due to tribocorrosion processes. 

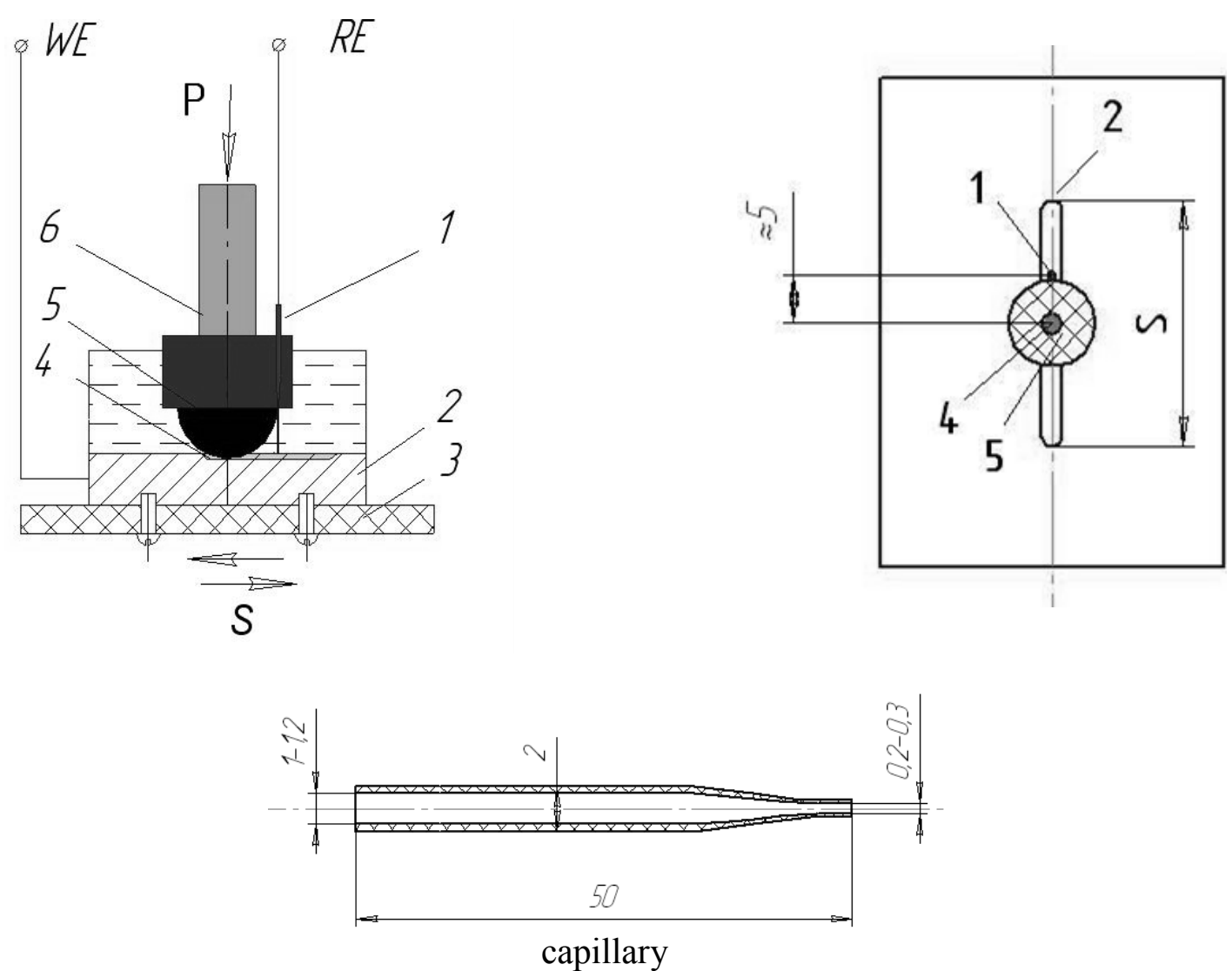

Figure 2. The scheme of open circuit potential measurements during tribocorrosion tests: 1 - capillary microprobe; 2 - sample; 3 - reciprocating stage; 4 - contact zone; 5 - corundum ball, 6 - indenter support with strain sensors; 7 - wear track; $R E-$ reference electrode; $W E$ - working electrode; $P$ - loading; $\mathrm{S}$ - direction of stage movement.

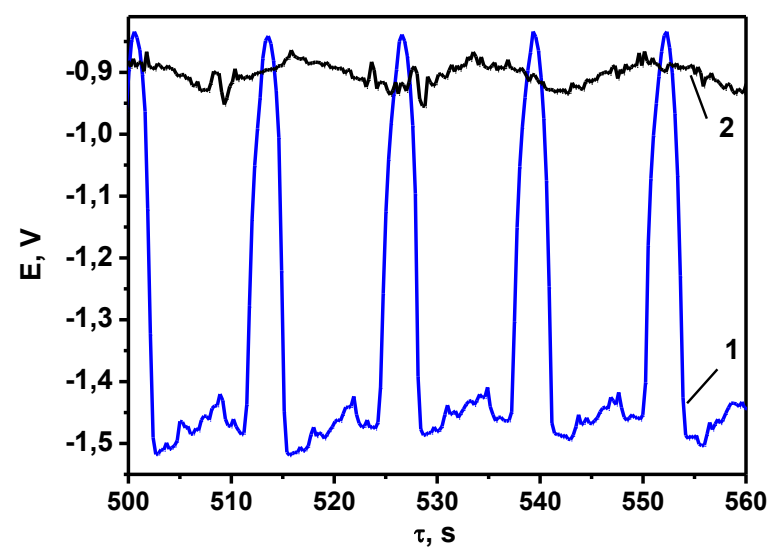

a

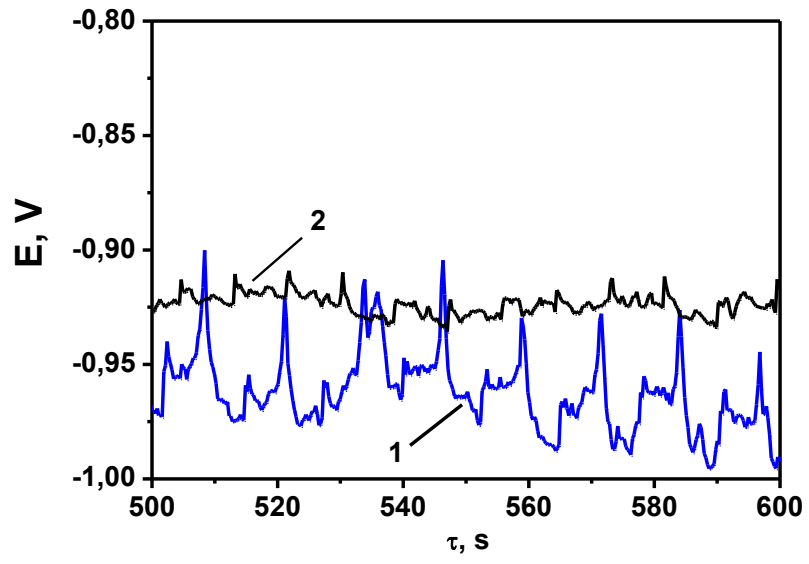

b

Figure 3. Local variations of the open circuit potential under friction conditions of aluminium alloy AA2024 in glycerin $(a)$ and in $3 \%$ solution of $\mathrm{NaCl}(b)$ measured by capillary microelectrode $(1)$ and with a classic saturated $\mathrm{Ag} / \mathrm{AgCl}$ reference electrode (2). Contact loading $-1 \mathrm{~N}$. 
The sudden shift of potential toward more positive values takes place at every microprobe move out from the track area and its contact with passive surface of sample (Fig. 3). This occurs during every cycle of friction and therefore cyclical nature of the changes is observed. The measurement of change in electrode potential by microprobe method is more informative, because clear correlation between tribopotential and friction moment exists (Fig. 4). Each local change (oscillation) of friction moment in the direction of growth correlates with the same oscillation of the electrode potential toward negative values.
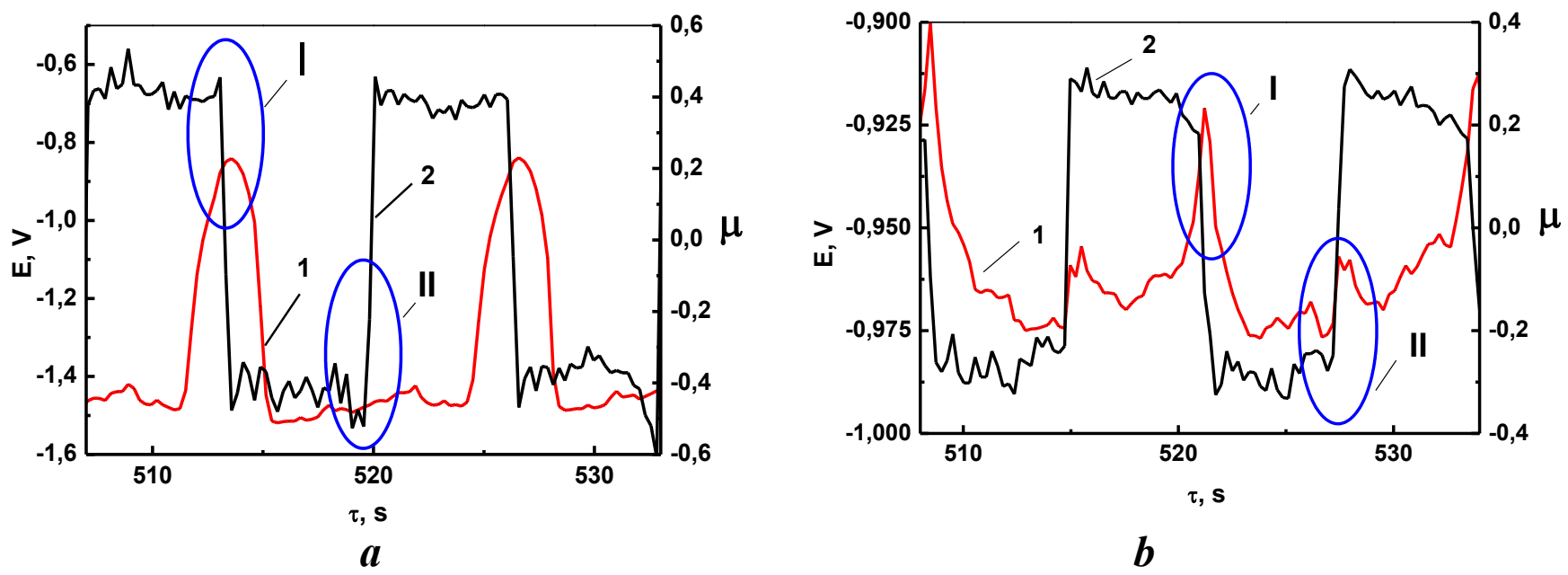

Figure 4. Local variations of electrode potentials (1) and friction coefficient $\mu$ (2) during tribocorosion tests of alloy AA2024 in glycerine $(a)$ and $3 \% \mathrm{NaCl}$ solution $(b)$ with use of $\mathrm{Ag} / \mathrm{AgCl}$ reference microprobe. Contact load $-1 \mathrm{~N}$.

It can be observed that electrode potential in glycerine significantly moves in the positive direction when the capillary microprobe goes out from wear track to an undamaged sample part with natural surface film (Fig. $4 a$, part I). This occurs in the moment of change in the movement direction. In area II, the movement direction change insignificantly affects the electrode potential, since formation of films due to destruction of glycerine and absence of passivator does not occur. Friction coefficient under these conditions reaches 0.4 . Unlike glycerol, in $3 \% \mathrm{NaCl}$ solution the movement of potential towards positive values is observed both in part I and in part II because formation of oxides and hydroxides occurs, reducing the friction coefficient to 0.3 (Fig. 4b).

\subsection{Polarization current change during tribocorrosion tests}

It is important to know the intensity of destruction and the approximate area of contact between the connected details in material tribocorrosion tests implemented using experimental schemes such as ball-plane, a cone-plane, the ball-ball and others where the sample/counter body contact area changes. Therefore, a method for determining the polarization current during tribocorrosion tests was proposed that makes determination of the volume of worn material much easier. 
The technique involves the analysis of changes in polarization current $\left(I_{\mathrm{pol}}\right)$ of a metal sample [8] during its tribocorrosion tests under polarization at corrosion potential $\left(E_{\mathrm{pol}}=\right.$ $\left.E_{\text {cor }}\right)$. The current in the absence of mechanical stress is zero, and a current flow will be caused mainly by work of a deformational galvanic couple: surface of wear track (anode) undamaged sample surface (cathode) not involved in friction. The ball in the "ball-plane" friction pair has significantly higher wear resistance compared with the sample material and its wear does not need to be considered. Time dependencies of polarization current obtained at two different loads are shown in Fig. 5. The sizes of corresponding wear tracks are determined from SEM images (Fig. 6). The relationship between polarization current $I_{\text {pol }}$ and track width $B$ can be obtained.

In our case, the depth of the wear track can be neglected because its value $h$ is much smaller than the ball diameter $(h<<D)$.

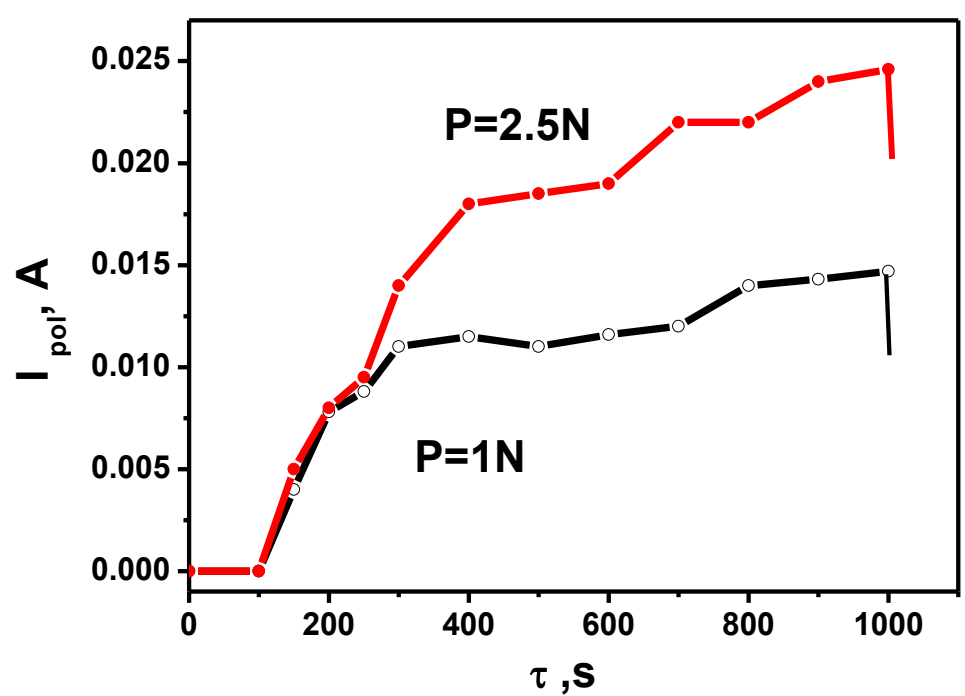

Figure 5. Time dependencies of alloy AA2024 polarization current in distilled water depending on applied load. (Counterbody: corrundum ball with $D=9 \mathrm{~mm}$ ).

It was found in experimental investigations that at $1 \mathrm{~N}$ load, the maximum polarization current and track width are $\sim 0.015 \mathrm{~A}$ and $\sim 0.19 \mathrm{~mm}$, correspondingly, and an empirical relationship was proposed:

$$
B=\alpha \cdot I_{\mathrm{pol}},
$$

where $\alpha$ is the coefficient of polarization current/track width ratio; $\alpha=12.67$ for the pair "AA2024 alloy $-\mathrm{Al}_{2} \mathrm{O}_{3}$ ball" in distilled water.

The correctness of the relationship between current and the geometrical parameter can be verified for $2.5 \mathrm{~N}$ load on indenter. In this case, the maximum current is $\sim 0.026 \mathrm{~A}$ and a track width of $0.329 \mathrm{~mm}$ is obtained by multiplying it by the coefficient of 12.67 . According to SEM data, this value is $0.325 \mathrm{~mm}$, and the calculation error is less than $2 \%$. The correctness of the method was confirmed at other loads. 


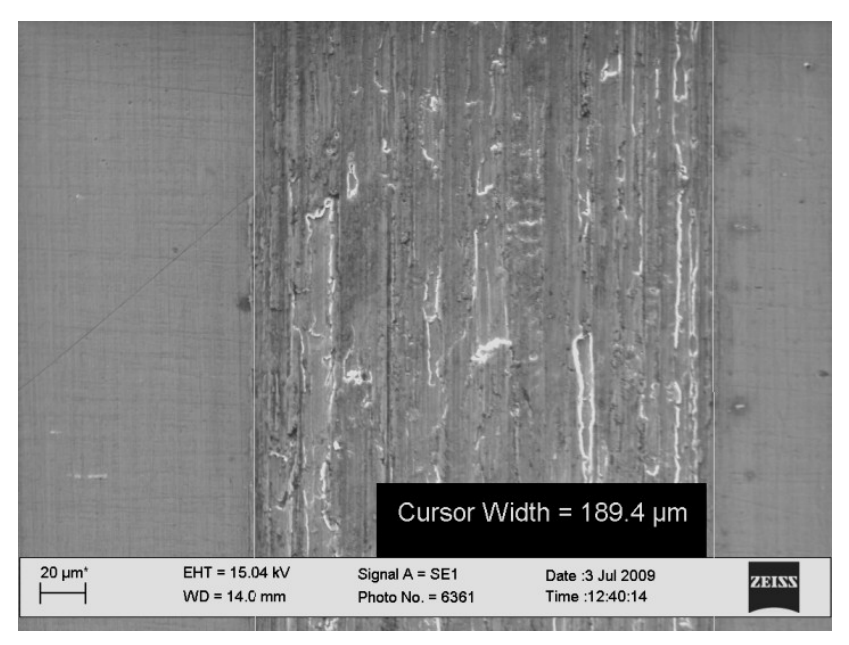

$a$

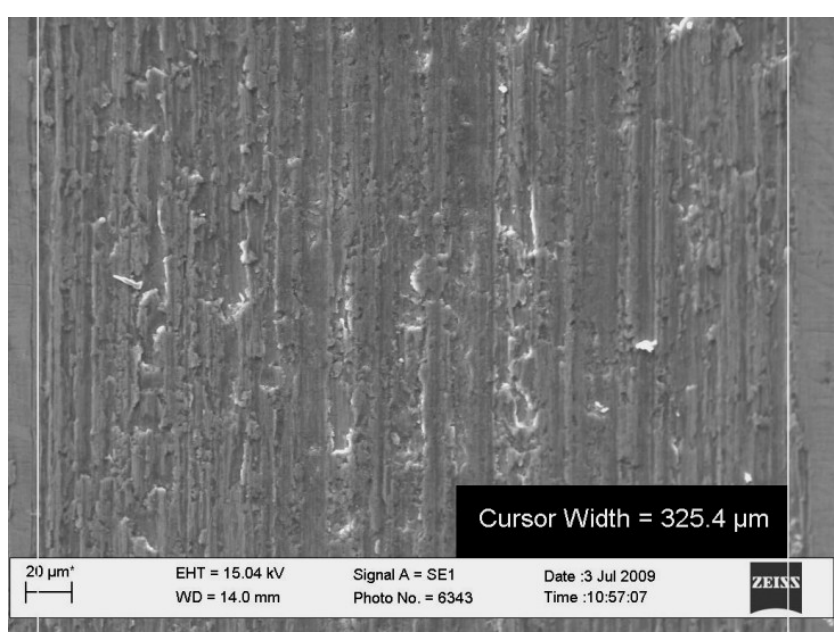

$b$

Figure 6. Surface topography of wear tracks on alloy AA2024 in distilled water at different loads on the corundum indenter.

Thus, a correlation has been established between polarization current and geometrical parameters of wear tracks for the same friction pair in a certain corrosion environment at various loads. This allows one to estimate the wear magnitude of the material based on polarization current values. The experimental approach was also applied to study the protection of an aluminium alloy from tribocorrosion with a phosphate inhibitor [9].

\section{Conclusions}

1. A capillary microprobe needs to be used for high precision measurements of corrosion potential during tribocorrosion tests in the vicinity of a friction contact, especially in poorly conducting medium. The difference in alloy AA2024 potentials in glycerine measured by the traditional method and by the proposed microcapillary method can be up to $600 \mathrm{mV}$.

2. Polarization current at potential $E_{\mathrm{pol}}=E_{\mathrm{cor}}$ can be used for a quantitative estimation of metal loss during tribocorrosion.

\section{References}

1. D. Landolt, M. Stemp and S. Mischle, Electrochim. Acta, 2001, 46, 3913.

2. D. Landolt, J. Phys. D: Appl. Phys., 2006, 39, 3121.

3. S. Mischler, Tribology Int., 2008, 41, 573.

4. M. Favero, P. Stadelmann and S. Mischler, J. Phys. D: Appl. Phys., 2006, 39, 3175.

5. J.-P. Celis, P. Pontiaux and F. Wenger, Wear, 2006, 261, 939.

6. A. Bidiville, M. Favero, P. Stadelmann and S. Mischler, Wear, 2007, 263, 207. 
7. V. Pokhmurskii, V. Dovhunyk, M. Student, H. Pokhmurska and V. Vynar, Surface Engineering, 2008, 1, 9.

8. V. Pokhmurskii, M. Khoma and V. Vynar, The European Corrosion Congres. Progress by Corrosion Control. "EUROCORR-2007”, Freiburg, Germany, 2007, CD-ROM, p. 7.

9. V. I. Pokhmurskii, I. M. Zin, V. A. Vynar et al., Corrosion Engineering, Science and Technology, 2012, 47, no. 3, 182. 